\title{
Observaciones sobre ingeniería civil en Cuba a propósito de su primer puente de hierro: una celosía Bollman de 1859 para el río Almendares de La Habana
}

\author{
Ignacio J. López Hérnández \\ Universidad de Almería \\ ignajh@ual.es
}

RESUMEN: En este artículo se documenta el desconocido puente de hierro sobre el río Almendares de La Habana, primera obra de esta naturaleza construida en Cuba en 1859 y debida al célebre ingeniero norteamericano Wendel Bollman. Hoy desaparecido, se dan a conocer el plano original y el expediente del proyecto, poniéndose en relación con otras iniciativas anteriores que buscaron implementar esta tecnología en el contexto de la actualización y desarrollo experimentados por las obras públicas en la Cuba decimonónica.

PALABRAS CLAVE: Cuba; Ingeniería; Obras Públicas; Puente; Transferencia tecnológica; Wendel Bollman.

\section{Notes on Civil Engineering in Cuba and its First Iron Bridge: an 1859 Bollman's Truss Bridge for the Almendares River in Havana}

\begin{abstract}
This article documents the now-defunct bridge over the Almendares river in Havana. By providing its administrative record and the original plan, designed by the American engineer Wendel Bollman, this paper proves that this structure was the first iron bridge built in Cuba in 1859. Furthermore, the bridge is studied as a significative example of the implementation of this kind of technology that supplied the public works of Cuba with the necessary means to its own development and updating.
\end{abstract}

KEYWORDS: Cuba; Engineering; Public Works; Bridge; Technology Transfer; Wendel Bollman.

Recibido: 25 de enero de 2020 / Aceptado: 11 de mayo de 2020.

Las obras públicas de la América española merecen valorarse hoy como uno de los principales recursos que posibilitaron la compleja gestión territorial de los amplios dominios de la corona en ultramar¹. A esta función básica de las obras públicas se agrega la de dinamizador comercial con la que fueron especialmente concebidas en el contexto decimonónico cubano. A pesar de los excelentes trabajos dedicados al ferrocarril (García y Zanetti, 1987; Moyano, 1991), aún se precisan estudios que documenten otros ámbitos de las obras públicas en los que igualmente reposó el éxito de la Cuba comercial azucarera, como fueron la construcción y habilitación de edificios y espacios dotacionales de todo tipo, así como faros, puertos, muelles, telégrafos, caminos vecinales, carreteras o puentes².

Recientemente ha sido posible documentar la estructura administrativa bajo la que se organizaron las obras públicas de la isla, coordinadas por corporaciones como la Junta Económica del Consulado de Agricultura y Comercio de La Habana, la Real Junta de Fomento de la Isla de Cuba (López, 2016: 483-508) y finalmente la Dirección de Obras Públicas de la Isla de Cuba (López, 2018: 323-335). Por su parte, ante la ausencia de ingenieros de caminos, no presentes hasta 1867 (Sáenz de Ridruejo, 2009: 314), el Cuerpo de Ingenieros Militares ejerció sus principales atribuciones facultativas, contraviniendo sus propias ordenanzas castrenses al integrarse incluso en la administración de estas corporaciones. A efectos fa-

Cómo citar este artículo: LÓPEZ HÉRNÁNDEZ, Ignacio J., "Observaciones sobre ingeniería civil en Cuba a propósito de su primer puente de hierro: una celosía Bollman de 1859 para el río Almendares de La Habana», Boletín de Arte-UMA, n. ${ }^{\circ}$ 41, Departamento de Historia del Arte, Universidad de Málaga, 2020, pp. 129-138, ISSN: 0211-8483, e-ISSN: 2695-415X, DOI: http://dx.doi.org/10.24310/BoLArte.2020.v41i.7574 
cultativos, a estos se unieron asimismo ingenieros civiles de origen extranjero que suplieron, por un lado, la necesidad de técnicos, y por otro, la falta de experiencia de los militares españoles en campos constructivos que les fueron formativamente ajenos como el ferrocarril. Ello dio lugar al contacto continuo de ingenieros formados en especialidades y escuelas distintas, lo que contribuyó a la creación de un canal fluido de transferencia técnica que redundó en la actualización tecnológica de sus construcciones, a veces con carácter precursor con respecto a la metrópoli.

\section{La construcción de puentes como vehículo de transferencia e innovación}

Uno de los ámbitos de las obras públicas que experimentó mayor desarrollo tecnológico fue el de la construcción de puentes. La máxima prioridad de la administración fue la creación de una red de comunicaciones que permitiera la conexión de múltiples plantaciones e ingenios con sus puertos exportadores. En esta labor, tanto para los trabajos en carreteras y caminos como para los del ferrocarril, la construcción de puentes tuvo gran protagonismo en atención a la compleja orografía de la isla, plagada de ríos o pequeños arroyos que en época de lluvias se convertían en potentes torrentes de agua cargados de maleza. Ello derivó en la constante ruina de estas estructuras, obligando a su continua reconstrucción, lo que dio lugar a un interesante debate técnico entre los ingenieros activos en la isla a fin de encontrar una solución adaptada. Así se registran numerosos proyectos, primero de ingenieros civiles franceses como Jules Sagebien o Arsène Lacarrière-Latour y más tarde de ingenieros militares españoles, en los que se empezaron a implementar técnicas y modelos de ingeniería europea de puentes desarrollados por autores como Muller (1769), Belidor (1754), Polonceau (1829), Gautier (1716), Rondelet (1830), Bruyère (1823), Gauthey (1813) y especialmente Perronet $(1788)^{3}$.

Fue en este contexto innovativo, permeable a soluciones externas, en el que se registran las primeras tentativas de construir un puente de hierro en la isla. Esta idea calaría con fuerza después del viaje que los comisionados del Consulado de La Habana Ramón Arozarena y Pedro Bauduy hicieron a Jamaica para estudiar su sistema de gestión agrícola y comercial, y en el que tuvieron la oportunidad de admirar el puente de hierro de Spanish Town construido en 1795 [1]. Así, entre las medidas que los comisionados instaban a adoptar para el fomento de la agricultura y el comercio de Cuba estaba la de incluir esta tipología de puentes para los futuros proyectos, dada su resistencia, ligereza y sencillez de montaje, así como por su capacidad de financiación y rápida amortización:

La idea que hay entre nosotros de que los puentes de hierro no pueden tener la misma solidez y duración que los demás puentes, (sobre todo debajo de los trópicos) ha hecho que los Comisionados pusiesen la mayor atención en el exámen que hicieron de un puente magnífico de hierro por donde pasaron [...] El puente de Spanish-Town, o Santiago de la Vega, fue hecho á costa del Gobierno en 1795, y el precio contratado en Inglaterra para su fundicion, fue de 1900 libras esterlinas, que son 8436 pesos. Es menester que vuestras señorías sepan que este puente es quizá el mas frecuentado de la isla [...] Desde el año de 1795 en que se hizo, hasta el de 1828 en que estamos, no ha necesitado la mas leve reparación, pues todas las piezas de respeto están todavía en el almacen. Puede juzgarse de su gran solidez, sabiendo que por el pasan todos los frutos de los contornos, y que el único medio de transportarlos es en carretas muy grandes de cuatro ruedas, conocidas con el nombre de wagon (Arozarena y Bauduy, 1828: 44-45).

No resulta extraño que por las mismas fechas en que se editaba la memoria de estos dos comisionados, el ingeniero Nicolás Campos proyectara un puente de hierro sobre el río Canímar de Matanzas (López, 2019: 307-312) proponiendo el característico sistema de armadura de aros tangentes a arco y tablero que utilizaba el ejemplo jamaicano, y que más tarde popularizarían a mayor escala los puentes de Carrousel en París y de Isabel II en Sevilla.

El elevado número de ríos que transcurren por los límites de la jurisdicción de Matanzas y su condición de una de las principales cabeceras agrícolas de la isla, hicieron de la proyección y construcción de puentes una de las principales vías de penetración de la nueva tecnología europea y norteamericana aplicada a estas estructuras. Así, al proyecto frustrado de Campos le siguieron otros similares, con igual éxito, como los ideados para el río San Juan de la misma ciudad 


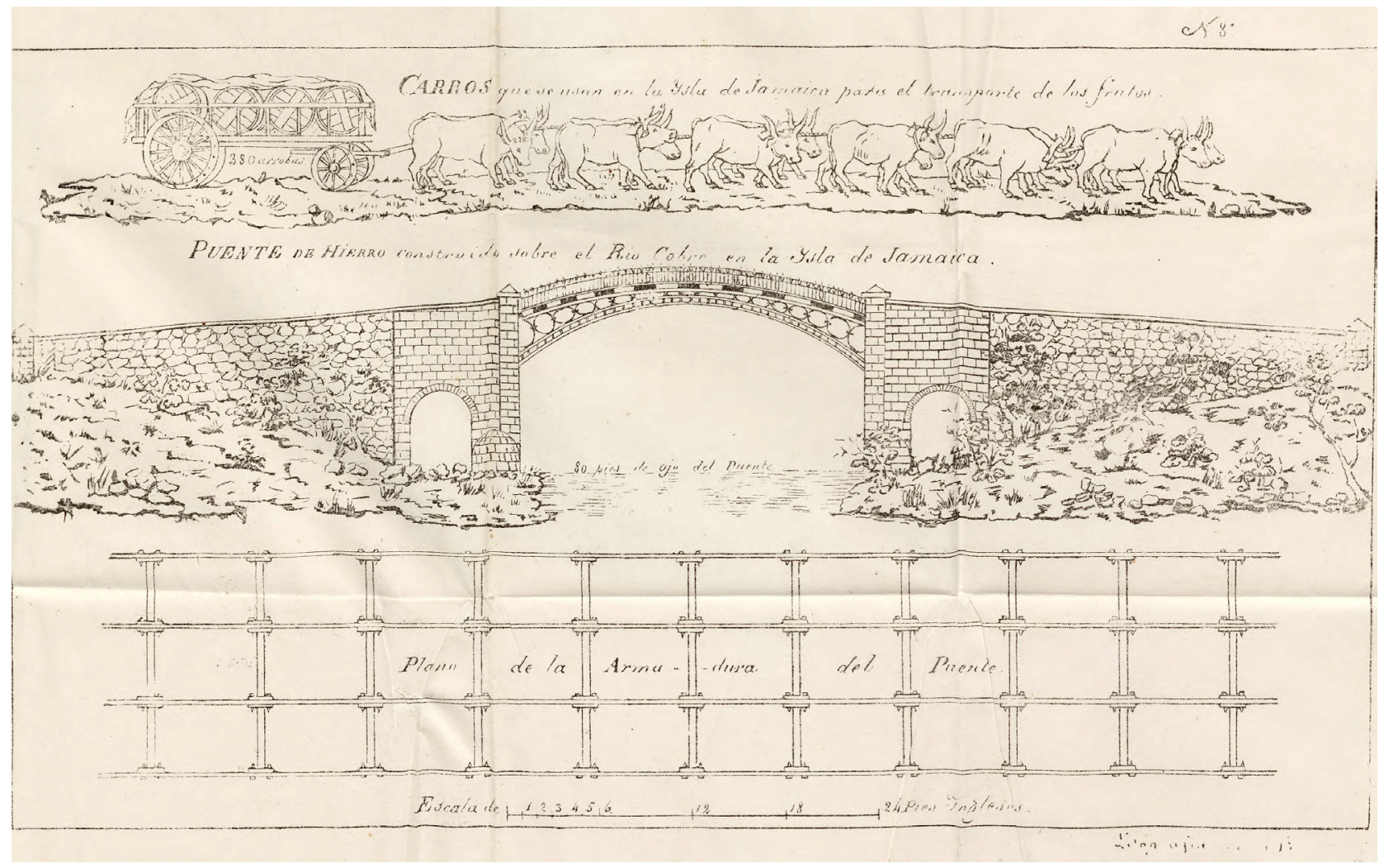

1. Puente de hierro construido sobre el río Cobre en la Ysla de Jamaica, en Arozarena y Bauduy, 1828, ilustración 8

por los ingenieros Jules Sagebien (1830) y Carlos Benítez (1845), de nuevo por influencia de la memoria de Arozarena y Bauduy dentro del mismo Consulado de La Habana. En el caso del proyecto del ingeniero francés se trató de un encargo doble hecho por el diputado del Consulado José de Ybarra para cubrir el paso del río en dos tramos distintos para los que Sagebien formó dos diseños siguiendo diferentes modelos y sistemas europeos (López, 2019: 236).

Por su parte, el comandante de ingenieros Carlos Benítez planteó, como alternativa a su proyecto de puente de cantería que se acabaría finalmente ejecutando, la utilización de una armadura de hierro. Sus planos y memoria muestran un puente de dos pilas y sendos estribos sobre los que apean arcos formados por estructuras metálicas segmentadas en módulos cuadrados arriostrados que permitían reducir considerablemente su flecha (López, 2014a: 279). Sin embargo, la experiencia constructiva de estos ingenieros no llegaba a ser suficiente, por lo que ellos mismos manifestaron dudas sobre la fiabilidad de estas estructuras, aún no plenamente desarrolladas tal y como demostraron las limitaciones de ejemplos como el de Coalbrookdale sobre el Severn o el de las Artes de París.

La solución definitiva acabó llegando poco después de la mano de la tecnología ferroviaria importada por ingenieros norteamericanos. Algunos, como Alfred Cruger, advirtieron de manera visionaria la necesidad de puentes que salvaran completamente las corrientes, para lo que, en la mayoría de los casos las estructuras en arco no eran suficientes. A ello comenzaron a dar respuesta los primeros puentes de celosía desarrollados en los Estados Unidos para las obras del ferrocarril, entre los años 20 y 30 del siglo XIX (Sutherland, 1998: 323-324 y 329). Patentadas bajo diversos modelos, sus armaduras de postes, viguetas y riostras permitían crear puentes apeados de estribo a estribo. Una de las patentes más exitosas fue la del ingeniero norteamericano William Howe, quien registró en 1840 una celosía de madera formada por una armadura de tramos cuadrados reforzados por diagonales inclinadas hacia el centro (Ritter, 1990: 263). Esta 


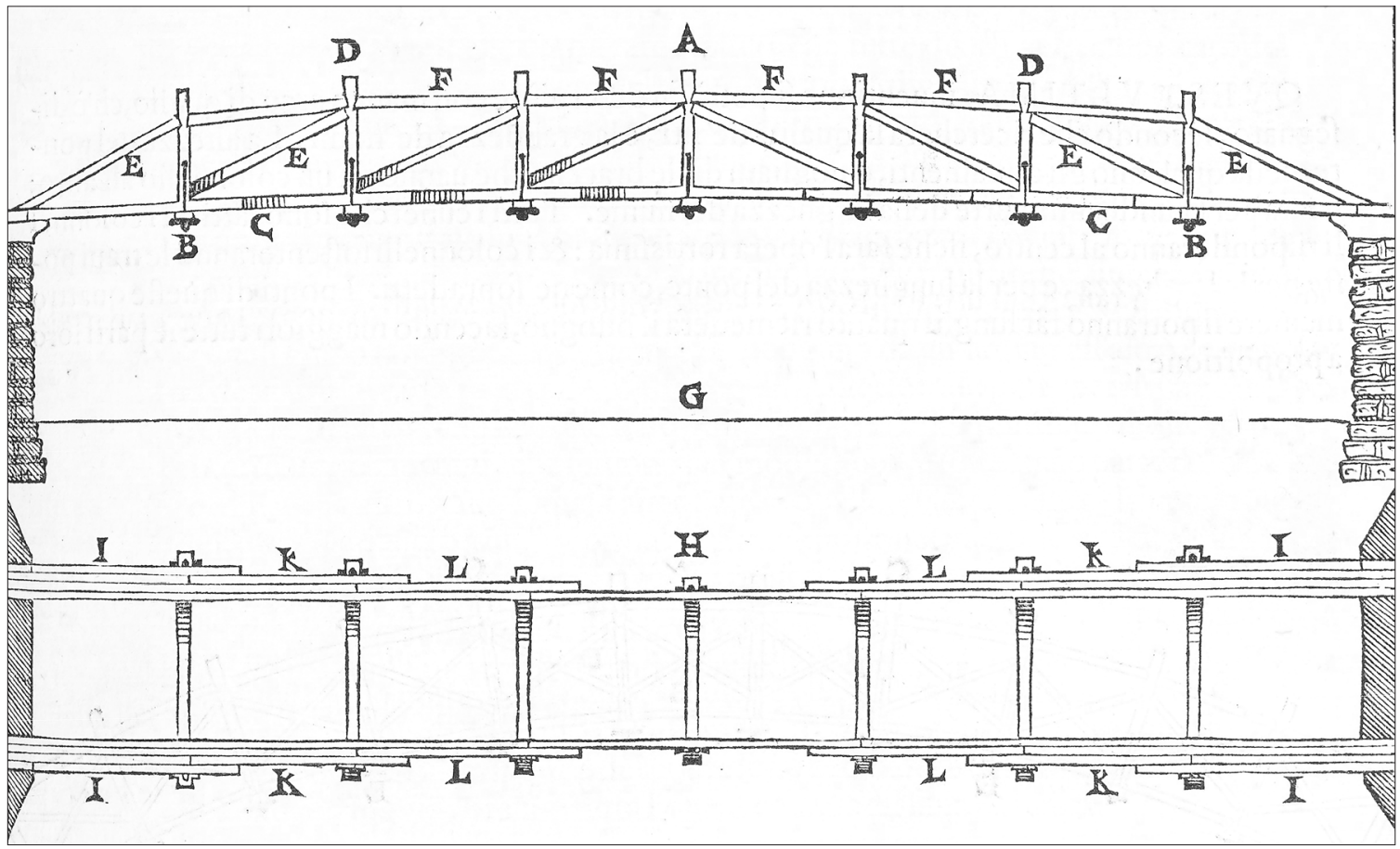

2. Dei ponti di legno, en Palladio, 1570: Libro III, pp. 16-17

sencilla estructura, muy difundida tanto en América como en Europa, no haría más que desarrollar una idea que ya Palladio propuso en 1570 para «ponti di legno senza porre altrimenti pali nel fiume» (1570: Libro III, pp. 16-17) [2]. Desde modelos sencillos como el de Howe, poco a poco comenzaron a introducirse piezas de metal con las que reforzar las estructuras, hasta que en 1845 el ingeniero de la Philadelphia and Reading Railroad, Richard B. Osborne, diseñó la primera celosía ferroviaria hecha completamente de hierro (Condit, 1968: 54).

La primera aproximación hacia esta tipología de puentes en Cuba tuvo lugar con la construcción en 1858 del puente de Martín Pérez en la carretera que conectaba La Habana con la población de La Gallega. Hoy desaparecido, de él se sabe a través de las memorias de la Dirección de Obras Públicas (Memoria, 1866: 42), que su estructura principal la conformaba una viga de celosía que seguía el sistema de William Howe ${ }^{4}$. El puente permitía el paso en dos sentidos separados al introducir tres cerchas de madera que cerraban los extremos y dividía su tránsito en dos carriles. Gracias a esta configuración se lograba salvar los 20 metros de luz que separaban sus dos estribos de mampostería. Aunque se trató de una estructura fundamentalmente de madera, fueron introducidas piezas de hierro con las que se conseguía un reforzamiento de la trama y una mayor capacidad de resistencia. Fue el primer paso para la definitiva introducción de este metal en esta tipología de puentes que tendría lugar, según ahora se documenta, en 1859 con el nuevo puente del río Almendares en La Habana.

\section{El puente de hierro sobre el río Almendares de Wendel Bollman}

La noticia de la existencia y construcción de esta obra, desconocida hasta ahora, trasciende por el hallazgo en el Archivo Histórico Nacional del expediente para la «Aprobación del proyecto de construcción de un puente sobre el río Almendares ${ }^{5}$. El proyecto contemplaba la construcción de un puente en la vecindad de Puentes Grandes, extramuros de La Habana, en el punto en el que el río se encontraba con el Arroyo Mordazo. Según consta por un plano de 1847 del 


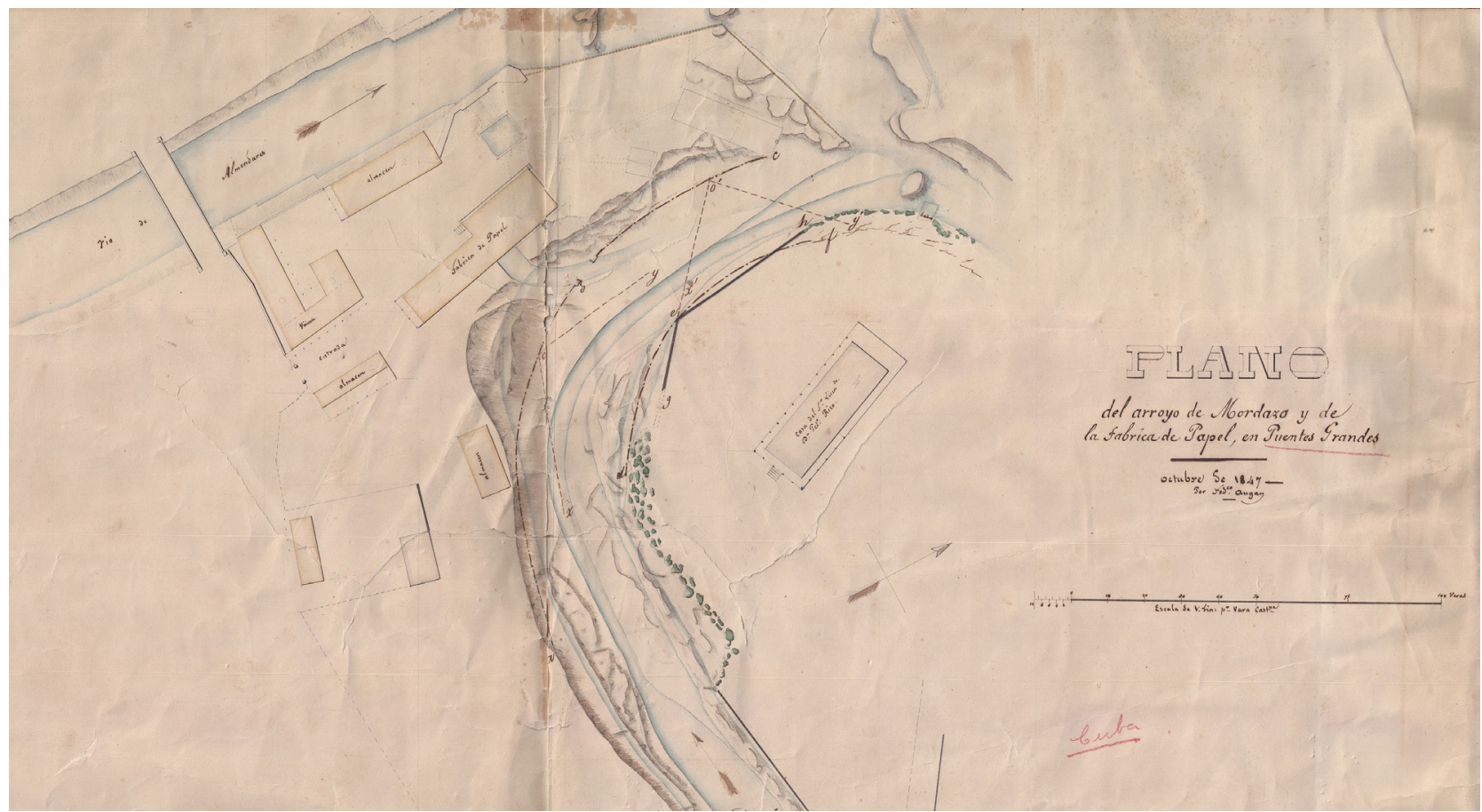

3. Plano del arroyo de Mordazo y de la fabrica de Papel, en Puentes Grandes, Federico Augan, octubre de 1847, AGMM, Cartoteca, CUB-79/07

arquitecto de la Academia de San Fernando Federico Augan [3], el puente primitivo cruzaba el río Almendares en las inmediaciones de una fábrica de papel, en un punto en el que su cauce entroncaba con el arroyo Mordazo ${ }^{6}$. La siguiente y última referencia a este puente es la citada por el director de obras públicas y comandante de ingenieros Francisco de Albear, quien lo reparó en torno a $1850^{7}$. Aunque no hay referencia al tipo de estructura, debió tratarse de un puente convencional de madera, según era práctica habitual y la recomendada por el propio Albear (García, 2007: 44). Esto mismo se deduce por la necesaria sustitución del puente apenas siete años más tarde, cuando se inicia el expediente conservado en Madrid, del que se cita, sin más información, que se hallaba en estado ruinoso.

El 2 de abril de 1857, el capitán general José Gutiérrez de la Concha solicitó a la sección de Fomento del Ministerio de la Guerra y Ultramar permiso y fondos para la construcción de un nuevo «puente oblicuo de madera con estribos de sillería según el sistema americano de Town». El proyecto del comandante de ingenieros Juan Modet fue aprobado por Real Orden de 4 de julio de 1857 a un precio de 20.197 pesos $^{8}$. Así, consta como el 12 de febrero de 1858 comenzaron los trabajos en la cimentación de los estribos ${ }^{9}$. Varios contratiempos relativos a la necesaria expropiación de terrenos y mutaciones en los puntos de cimentación elegidos para los estribos, acabaron lastrando el proyecto, que sufrió una nueva modificación que incrementó su presupuesto hasta los 32.603 pesos $^{10}$. Superados los problemas en los estribos, se presentaron otros de abastecimiento de maderas, por lo que la Dirección de Obras Públicas resolvió sustituir la estructura original por una de hierro. A ello respondió un nuevo proyecto de Juan Modet en octubre de 1858 con el que se volvía a aumentar el presupuesto hasta los 35.863 pesos $^{11}$. En sus planos ya se detallaba la nueva configuración de los estribos, ligeramente oblicuos, en los que apoyaría una viga de celosía con sección en «» que seguía el mismo sistema constructivo de Town previsto para el de madera ${ }^{12}$.

El proyecto de Modet no prosperó, prefiriéndose contar con otros especialistas más versados en aquel nuevo tipo de estructuras. De esta forma se decidió encargar la construcción del puente a una compañía norteamericana, cuyo 


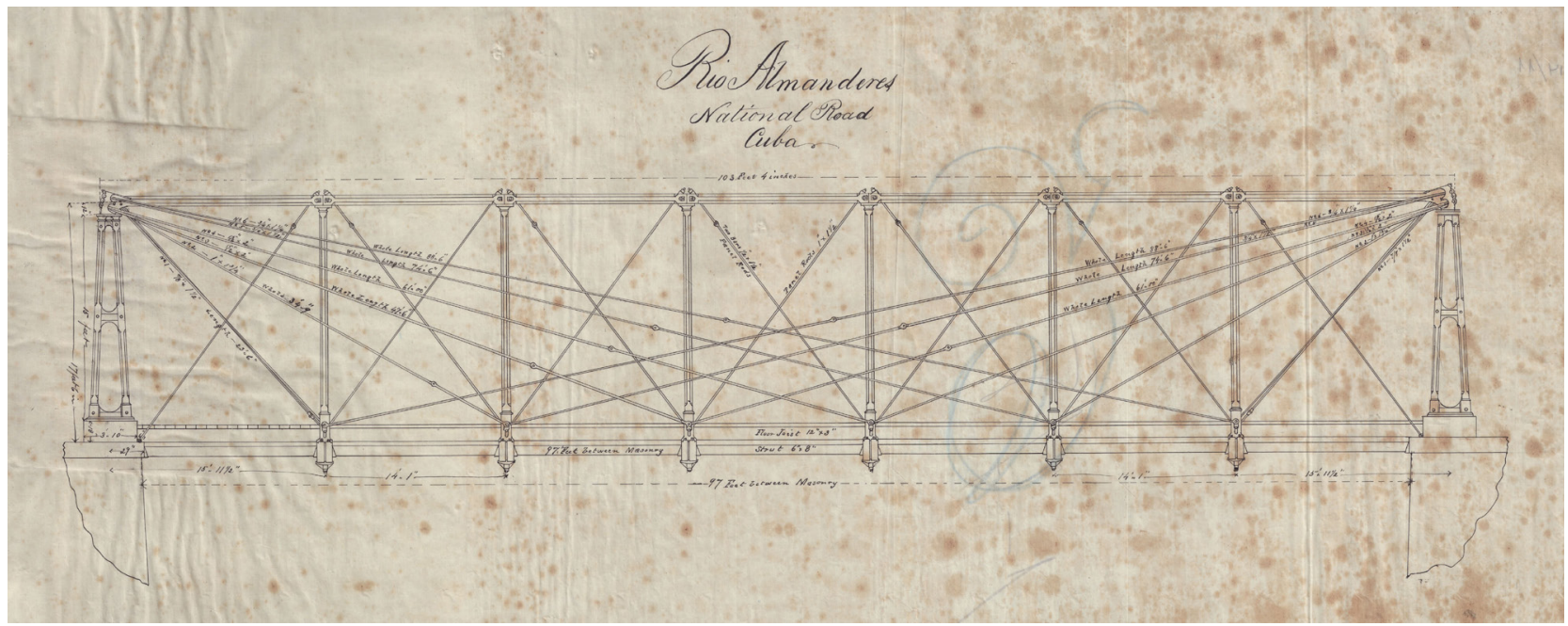

4. Rio Almanderes National Road Cuba, Wendel Bollman, h. 1859, AGMM, Cartoteca, CUB-72/13 (detalle 1)

armazón habría de fletarse hasta La Habana para su montaje sobre los estribos en construcción. Para ello, el ingeniero de la compañía del Ferrocarril de La Habana M. O. Davidson remitió una carta de recomendación a la compañía Bollman de Baltimore en la que se especificaban las líneas generales de un puente de 92 pies ingleses de luz por 25 de anchura (28 por $7,62 \mathrm{~m})^{13}$.

Para coordinar el encargo se comisionó en Nueva York al ingeniero militar Juan Campuzano que habría de tratar directamente con Wendel Bollman y con los comerciantes Pesant y Hermanos, que harían luego de intermediarios ${ }^{14}$. Por vía de estos últimos, Bollman envió un plano según las especificaciones acordadas por un valor de 9.600 pesos, de los que se excluía el flete. Se incluían además refuerzos en su estructura a fin de que aguantara con solvencia el peso de la artillería pesada que se conducía por aquella carretera hasta las fortificaciones de la ciudad. Por último, la instalación de la obra sería sencilla, necesitando tan solo la presencia de un carpintero y una docena de trabajadores de fuerza ${ }^{15}$. El proyecto fue evaluado como satisfactorio por el ingeniero Juan Modet, si bien trasladó la necesidad de aplicar algunas modificaciones al proyecto final en atención a las variaciones sufridas por los estribos en los trabajos de terminación. El cambio más notable fue el de la ampliación de la luz del puente desde los 92 pies iniciales hasta los 97 (29,56 m), a lo que se añadía la colocación del escudo de Isabel II enca- bezando la entrada; la instalación de un antepecho de madera independiente de la armadura de hierro; y la remisión de cuatro bloques de granito sobre los que debía apoyar las «torres de hierro" que flanqueaban la entrada del puente. Con las correspondientes variaciones sobre el presupuesto, se solicitó a la compañía Bollman que formalizara el contrato en las condiciones acordadas previamente ${ }^{16}$.

En este punto, y ante la necesidad de arbitrar la compra, el capitán general Gutiérrez de la Concha solicitó la libranza de un anticipo de 15.666 pesos correspondientes a los aumentos sobre el presupuesto aprobado por la reina en 1857, para lo que debía contar con nueva aprobación real. Este último trámite es el que dio origen al expediente del que emanan todas estas noticias, motivo por el que, tras la Real Orden de 8 de marzo de 1859 en la que se aprobaba la modificación ${ }^{17}$, cesó cualquier información relativa al avance de los trabajos. Sin embargo, suple esta laguna un plano conservado en la Cartoteca del Archivo General Militar de Madrid que se identifica como Rio Almanderes (sic) National Road Cuba ${ }^{18}$. Aunque toda información escrita sobre la obra se limita al título, se trata sin duda del plano final remitido por Bollman, pues en él constan todas las medidas de altura y anchura acordadas, incluida la nueva luz de 97 pies [4]. A ello se agregan otros detalles como el escudo real sobre la vigueta de acceso al puente, donde asimismo aparece la fecha de 1859 [5]. 
Por lo demás, el plano describe con detalle un modelo canónico de la celosía desarrollada por Wendel Bollman en 1852, una de las primeras enteramente de hierro en ser patentada y comercializada (Berlow, 2015: 210) [6]. Se trataba de una viga de celosía que combinaba el hierro forjado y fundido formando tramos cuadrados arriostrados por barras de hierro que formaban aspas. La principal innovación de la celosía Bollman, presente en el proyecto habanero, fue la incorporación de nuevas riostras formadas por largas barras metálicas que, partiendo del remate de cada una de las cuatro «torres» o postes que flanqueaban los accesos, reforzaban cada uno de los tramos de la armadura de manera similar a como funciona el sistema de los puentes colgantes. Determinaba así una característica malla entrelazada de líneas diagonales que cruzaban de uno a otro lado del puente.

\section{Conclusiones: el primer puente de hierro de Cuba}

Aunque el hallazgo del plano del proyecto tampoco prueba su ejecución final, sí se puede confirmar la compra e instalación del puente gracias a una cita contenida en una memoria manuscrita conservada en la Biblioteca Nacional de España en la que el ingeniero Juan Campuzano da cuenta de las obras públicas ejecutadas en la isla de Cuba entre 1795 y 1859. Así, en referencia a los trabajos en el tramo de la Carretera del Oeste que pasaba por Puentes Grandes, dice del nuevo puente que se estaba construyendo que «se compone de un tablero de madera suspendido por 2 armaduras de hierro fundido y forjado, cuyas extremidades descansan en columnas y estas en estribos de canteria. Su luz es de 96 pies ingleses ó sean 29,26 metros lineales»'19. Esta información permite, asimismo, valorar otra cita más breve en una memoria posterior en la que se expresa que «en 3 de octubre de 1859 se abrió al tránsito público el puente de hierro sobre el rio Almendares, de 29 metros de luz, cuya reseña se hizo en la Memoria anterior ${ }^{20}$.

Desafortunadamente se trata de una obra hoy perdida, de la que apenas se tienen más referencias que las citadas. Por diversos planos, formados entre 1859 y 1898, se tiene constancia de la existencia en aquel punto de un puente ${ }^{21}$, hasta que fue sustituido entre 1927 y 1931 por otro de hormigón con motivo de la construcción de la Carretera Central

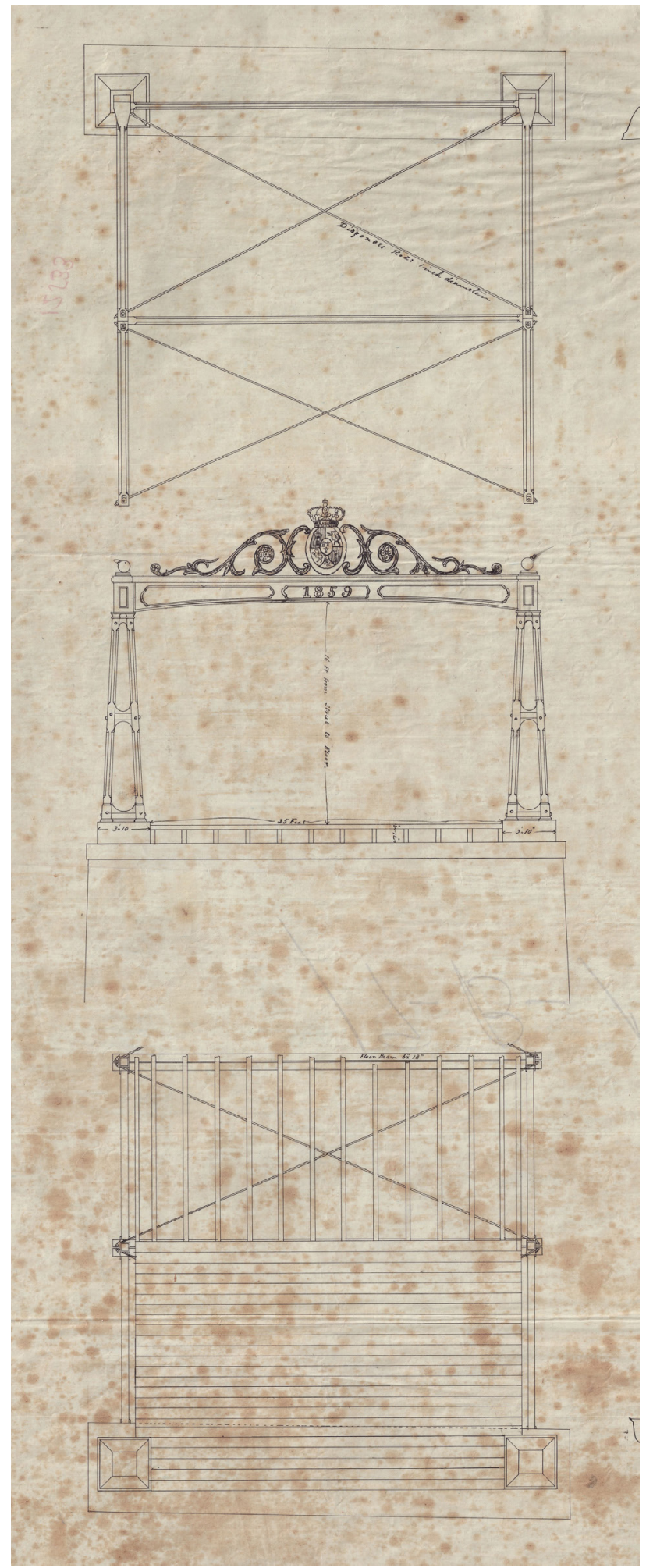

5. Rio Almanderes National Road Cuba, Wendel Bollman, h. 1859, AGMM, Cartoteca, CUB-72/13 (detalle 2) 


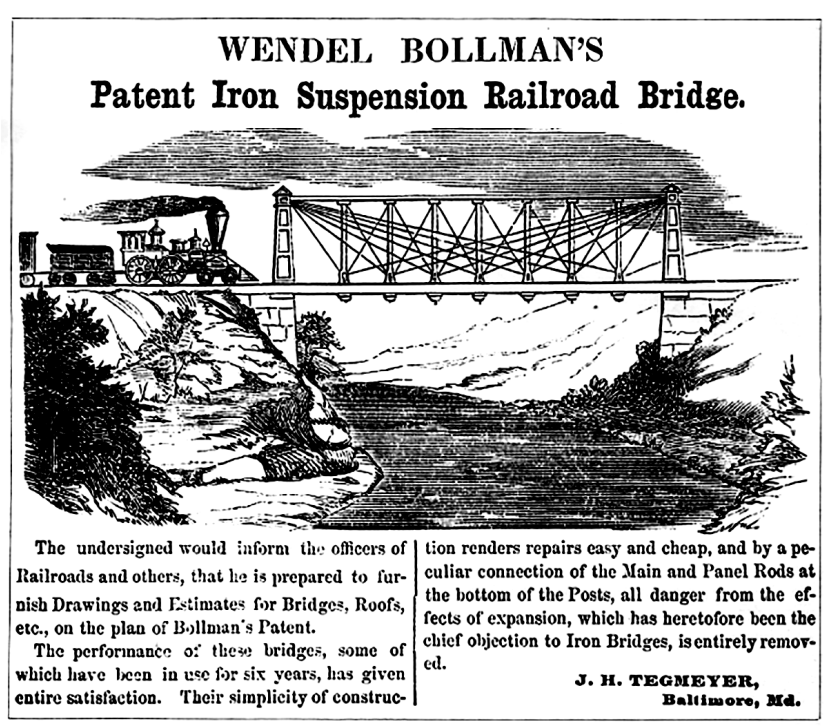

6. Wendel Bollman's Patent Iron

Suspension Railroad Bridge. Anuncio

en Railroad Advocate, 1855. Project

Gutenberg Free License

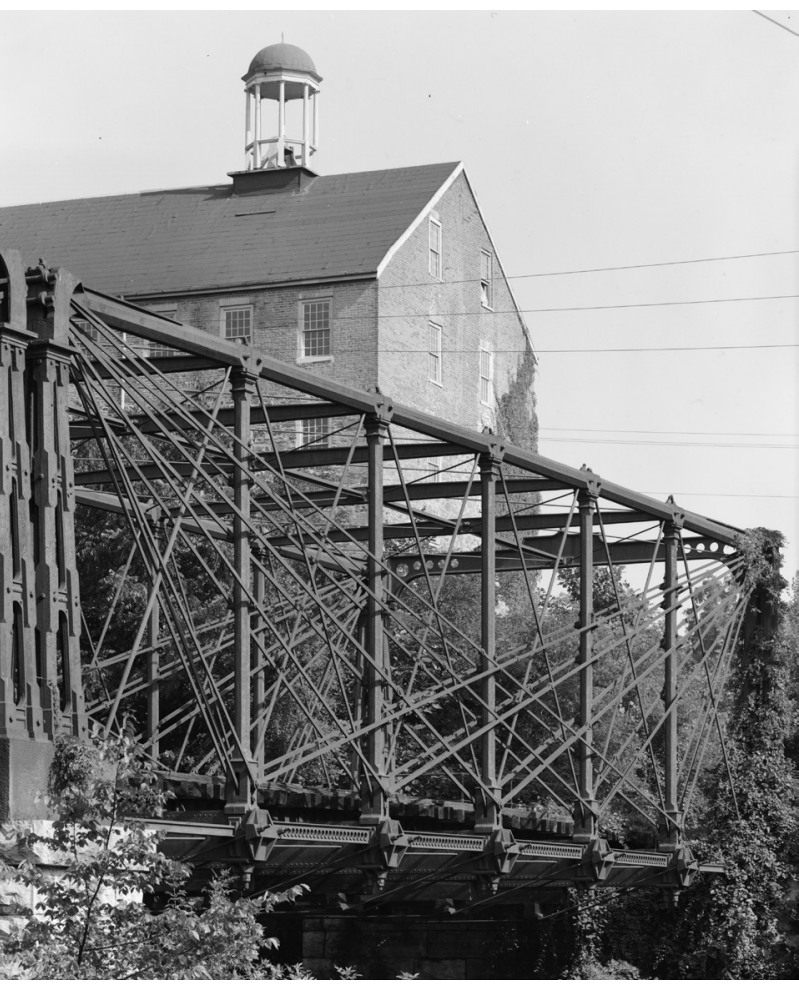

de Cuba. Con todo, se verifica por primera vez la existencia en la isla de uno de los sistemas de puentes más innovadores del momento, pudiéndose considerar asimismo como una de las primeras implantaciones del sistema de Bollman fuera de los Estados Unidos 22 . En este país hoy tan solo se conserva el de Savage, Maryland, construido diez años después del habanero bajo líneas compositivas muy similares a este [7]. Por último, estas noticias permiten adelantar 19 años la fecha de construcción del primer puente de hierro en Cuba, considerado como tal hasta ahora el de la Concordia de Matanzas, obra de 1878 del ingeniero y arquitecto Pedro Celestino del Pandal (García, 2009: 163; González y Recondo, 2011: 27-31).

Al margen de estas consideraciones, la obra también merece valorarse como un buen ejemplo de la abierta disposición de la administración cubana a nuevas soluciones que venían del exterior. La experiencia del ferrocarril y la apertura de su comercio posibilitaron un servicio de obras públicas permeable a soluciones aún experimentales, con cierto grado de independencia con respecto a la península. Así se entiende la novedad que supuso no solo en Cuba, sino en ámbito nacional la introducción de la celosía Bollman, en fechas en las que solo contados ejemplos en la península, caso del puente sobre el Besós de 1850 (Fernández y Sáenz, 2011: 489), llegaron a anticiparse a esta tecnología.

7. General view of truss on east side of bridge, Baltimore \& Ohio Railroad, Savage, Howard County. Fotografía de William E. Barrett, 1970. Library of Congress, Prints \& Photographs Division, HAER MD, 14-SAV,1-6 


\section{Notas}

1 Las dos principales obras sobre este particular se deben a González (1992), Laorden (2008) y Colomar y Sánchez de Mora (2019).

2 Sobre el papel del ingeniero militar en las obras civiles de Cuba consúltese López (2014b).

3 Para saber acerca de la aplicación de estos tratados en ejemplos de ingeniería de puentes en Cuba véase López, 2019: $207-322$.

4 Memoria General de las Obras Publicas en la isla de Cuba, Juan Campuzano, 1859, Biblioteca Nacional de España, Mss/13949, fol. 61r.

5 Aprobación del proyecto de construcción de un puente sobre el río Almendares, Archivo Histórico Nacional (AHN), Ultramar, legajo 63, exp. 16.

6 Plano del arroyo de Mordazo y de la fabrica de Papel, en Puentes Grandes, octubre de 1847, Archivo General Militar de Madrid (AGMM), Cartoteca, CUB-79/07.

7 Memoria del Teniente Coronel Comandante de Yngenieros D. Francisco Alvear encargado de las obras de la Junta de Fomento, 5 de noviembre de 1850, en AHN, Ultramar, legajo 31, exp. 17, s/f.

8 Comunicación del jefe de sección del Ministerio de la Guerra y Ultramar Gabriel Enríquez, 22 de junio de 1857, en AHN, Ultramar, legajo 63, exp. 16, Aprobación del proyecto..., pp. 5-6. Nota: para la paginación de este expediente se ha acudido al orden del volumen facticio generado digitalmente, debido a que no se encuentra foliado.

9 El capitán pedáneo de Puentes Grandes al brigadier gobernador político de La Habana, 12 de febrero de 1858, en AHN, Ultramar, legajo 63, exp. 16, Aprobación del proyecto..., pp. 73-75.

10 Proyecto de puente de madera sobre el río Almendares en Puentes Grandes, Juan Modet, 10 de mayo de 1858, en AHN, Ultramar, legajo 63, exp. 16, Aprobación del proyecto..., pp. 142-147.

11 Presupuesto para la construcción de un puente de hierro, Juan Modet, octubre de 1858, en AHN, Ultramar, legajo 63, exp. 16, Aprobación del proyecto..., pp. 316-322.

12 Puente de hierro sobre el Río Almendares en el Pueblo de Puentes-Grandes, Juan Modet, 12 de octubre de 1858, AHN, Ultramar, MPD. 1525.

13 Carta de M. O. Davidson a Wendel Bollman y Asociados, agosto de 1854, en AHN, Ultramar, legajo 63, exp. 16, Aprobación del proyecto..., pp. 169-170.

14 Vogel ya documentó la presencia de la compañía Bollman en algunos trabajos ferroviarios de la Compañía de La Habana a principios de los años sesenta, aunque sin mención alguna acerca de la obra que aquí se aborda, que bien pudo convertirse en punto de partida de encargos posteriores (1966: 92).

15 Juan Campuzano al director de Obras Públicas, en cartas de 2 y 13 de septiembre de 1858, en AHN, Ultramar, legajo 63, exp. 16, Aprobación del proyecto..., pp. 176-177.

16 Juan Campuzano a Bollman y Compañía, 28 de septiembre de 1858, en AHN, Ultramar, legajo 63, exp. 16, Aprobación del proyecto..., pp. 314-316.

17 Real Orden de 8 de marzo de 1859 por la que se aprueba el proyecto de puente de hierro sobre el río Almendares, en AHN, Ultramar, legajo 63, exp. 16, Aprobación del proyecto..., pp. 487-487.

18 Rio Almanderes National Road Cuba, Wendel Bollman, h. 1859, AGMM, Cartoteca, CUB-72/13.

19 Memoria General de las Obras Publicas..., doc. cit., fols. 40v-41r.

20 Memoria, 1866: 42.

21 Véase AGMM, Cartoteca, CUB-57/07; CUB-116/07; CUB-124/18; Centro Geográfico del Ejército, Cartoteca, Ar.j-t.6-c.1-139; Ar.j-t.5-c.3-49; Ar.j-t.6-c.1-144.

22 Según Vogel, la primera obra de Bollman documentada fuera de Estados Unidos fue un puente construido en Chile sobre el río Angustura para la compañía chilena de ferrocarriles en 1860 (Vogel, 1966: 100). Con esta noticia se adelanta un año la salida del modelo de Bollman de los Estados Unidos.

\section{Bibliografía}

AROZARENA, Ramón de y BAUDUY, Pedro (1828), Informe presentado a la Junta de Gobierno del Real Consulado de la Siempre Fiel Isla de Cuba, sobre el estado de la agricultura, y elaboracion y beneficio de los frutos coloniales en la de Jamayca. Imprenta fraternal de los Díaz de Castro, La Habana.

BELIDOR, Bernard Forest de (1754), La science des ingenieurs dans la conduite des travaux de fortification et d'architecture civile, Perre Gosse Junior, La Haya.

BERLOW, Lawrence (2015), Reference Guide to Famous Engineering Landmarks of the World, Fitzroy Dearborn Publishers, Chicago-Londres. BRUYERE, Louis (1823), Études relatives à l'art des constructions, vols. I-II, Bance Aîné, París.

COLOMAR ALBÁJAR, María Antonia, SÁNCHEZ DE MORA Y ANDRÉS, Ignacio (coords.) (2019), Cuatro siglos de ingeniería española en Ultramar. Siglos XVI-XIX, ASICA, Sevilla.

CONDIT, Carl W. (1968), American building: materials and techniques from the first colonial settlements to the present, University of Chicago Press, Chicago.

FERNÁNDEZ TROYANO, Leonardo y SÁENZ SANZ, Amaya (2011), "Los puentes. Materiales, estructuras y patrimonio", en SILVA SUÁREZ, Manuel (ed.), El Ochocientos: de los lenguajes al patrimonio, Real Academia de Ingeniería, Institución Fernando el Católico, Prensas Universitarias de Zaragoza, Zaragoza, pp. 451-498. 
GARCÍA BLANCO, Rolando (2007), Francisco de Albear. Un genio cubano universal, Editorial Científico-Técnica, La Habana.

GARCÍA SANTANA, Alicia (2009), Matanzas, la Atenas de Cuba, Polymita, Ciudad de Guatemala.

GARCÍA, Alejandro y ZANETTI, Óscar (1987), Caminos para el Azúcar, Editorial de Ciencias Sociales, La Habana.

GAUTHEY, Émiland (1813), Traité de la construction des Ponts. Vols. I-II, Chez Firmin Didot, París.

GONZÁLEZ ARESTUCHE, Luis Roberto y RECONDO PÉREZ, Ramón Félix (2011), Puentes de Matanzas, Editorial Matanzas, Matanzas.

GONZÁLEZ TASCÓN, Ignacio (1992), Ingeniería española en ultramar (siglos XVI-XIX), CEHOPU, Madrid.

LAORDEN RAMOS, Carlos (2008), Obra civil en Ultramar del Real Cuerpo de Ingenieros, Ministerio de Defensa, Madrid.

- (2012), «Obras civiles en América del Arma de Ingenieros, Revista de Historia Militar, n. ${ }^{\circ}$ Extraordinario, pp. 137-154.

LÓPEZ HERNÁNDEZ, Ignacio J. (2014a), "Carlos Benítez y los puentes de la ciudad cubana de Matanzas en 1849», Laboratorio de Arte, n. ${ }^{\circ} 26$, pp. 301-313.

- (2014b), «Antonio Montenegro y las funciones del ingeniero militar en la Cuba del Ochocientos», Quiroga. Revista de Patrimonio Iberoamericano, n. ${ }^{\circ}$ 5, pp. 74-84.

- (2016), «El Cuerpo de Ingenieros Militares y la Real Junta de Fomento de la Isla de Cuba. Obras públicas entre 1832 y 1854», Espacio, Tiempo y Forma, Serie VII, Historia del Arte, n. ${ }^{\circ} 4$, pp. 483-508.

- (2018), «El Cuerpo de Ingenieros Militares y la Dirección de Obras Públicas de la Isla de Cuba (1854-1867)», en W. AA., La formación artística: creadores, historiadores, espectadores. Vol. I, Editorial de la Universidad de Cantabria, Santander, pp. 323-335.

- (2019), Ingeniería e Ingenieros en Matanzas. Defensa y obras públicas entre 1693 y 1868, Athenaica, Sevilla,

Memoria sobre el progreso de las Obras Públicas en la Isla de Cuba desde el $1^{\circ}$ de enero de 1859 a fin de junio de 1865 (1866), Imprenta del Gobierno y Capitanía General, La Habana.

MOYANO BAZZANI, Eduardo (1991), La nueva frontera del azúcar: el ferrocarril y la economía cubana del siglo XIX, CSIC, Madrid.

MULLER, John (1769), Tratado de Fortificación ó Arte de construir los edificios militares y civiles, vols. I-II, Thomas Piferrer, Barcelona. Traducción de Miguel Sánchez Taramas.

PALLADIO, Andrea (1570), I quattro libri dell'architettura di Andrea Palladio, Domenico de' Franceschi, Venecia.

PERRONET, Jean-Rodolphe (1788), Description des projets et de la construction des Ponts de Neuilli, de Mantes, d'Orléans, de Louis XVI, etc, Françoix-Ambroise Didot, París.

POLONCEAU, Antoine-Rémy (1829), Notice sur quelques parties de travaux hydrauliques, Chez Carillan-Goeury, Libreire des ponts et chausséss, París.

RITTER, Michael A. (1990), Timber Bridges: Design, Construction, Inspection, and Maintenance, U.S. Department of Agriculture, Washington DC.

RONDELET, Jean (1830), Traité théorique et pratique de l'Art de Batir, Chez M. A. Rondelet fils, París.

SÁENZ RIDRUEJO, Fernando (2009), «Ingenieros de caminos en Puerto Rico, 1866-1898», Anuario de Estudios Atlánticos, n. 55, pp. 311341.

SUTHERLAND, R. J. M. (1998), Structural Iron 1750-1850, Routledge, Londres.

VOGEL, Robert M. (1966), «The engineering contribution of Wendel Bollman», Smithsonian Institution United States National Museum Bulletin, n. ${ }^{\circ} 240$, pp. $77-104$. 